\title{
Comparison of Visual Performance and Patient Satisfaction After Multifocal Intraocular Lens Implantation and During Multifocal Contact Lens Wear After Monofocal Intraocular Lens Implantation: A Pilot Study
}

Kazutaka Kamiya (D) - Kei Iijima • Takahiro Hiraoka • Keita Takada •

Yosai Mori · Kazunori Miyata

Received: August 20, 2021 / Accepted: October 15, 2021 / Published online: October 27, 2021

(C) The Author(s) 2021

\section{ABSTRACT}

Purpose: To compare the visual performance and patient satisfaction in multifocal intraocular lens (IOL)-implanted eyes and multifocal contact lens (CL)-wearing eyes undergoing monofocal IOL implantation.

Methods: We retrospectively assessed visual acuity at all distances $(0.3,0.5,0.7,1$, and $5 \mathrm{~m})$, contrast sensitivity function, patient satisfaction score, and the rate of spectacle independence in the multifocal IOL and multifocal CL groups.

Results: Binocular visual acuity at $0.3,0.5,0.7$, 1 , and $5 \mathrm{~m}$ was $0.05 \pm 0.11,-0.02 \pm 0.09$, $-0.02 \pm 0.09, \quad-0.02 \pm 0.09, \quad$ and $-0.04 \pm 0.07$, respectively, in the multifocal IOL group and $0.25 \pm 0.13,0.04 \pm 0.10$,

K. Kamiya ( $($ )

Visual Physiology, School of Allied Health Sciences, Kitasato University, 1-15-1 Kitasato, Minami,

Sagamihara, Kanagawa 252-0373, Japan

e-mail: kamiyak-tky@umin.ac.jp

K. Iijima

Department of Ophthalmology, School of Medicine, Kitasato University, Kanagawa, Japan

T. Hiraoka

Department of Ophthalmology, School of Medicine,

Tsukuba University, Ibaraki, Japan

K. Takada · Y. Mori · K. Miyata

Department of Ophthalmology, Miyata Eye

Hospital, Miyazaki, Japan
$0.01 \pm 0.09, \quad-0.01 \pm 0.07$, and $0.00 \pm 0.08$, respectively, in the multifocal CL group. We found significant differences in visual acuity at all distances. The area under the log contrast sensitivity function was $1.32 \pm 0.14$ and $1.33 \pm 0.16$ in the multifocal IOL and CL groups, respectively $(p=0.444)$. The patient satisfaction score for overall vision was $80.2 \pm 20.6$ and $82.9 \pm 10.5(p=0.889)$, and the rate of spectacle independence was $23.4 \%$ and $41.7 \%$ in the multifocal IOL and CL groups, respectively $(p=0.277)$.

Conclusions: According to our experience, both multifocal IOLs and CLs provided good near-to-distance binocular vision and subsequent high patient satisfaction in daily activities with acceptable contrast sensitivity, suggesting their viability for presbyopic correction in elderly subjects.

Keywords: Multifocal intraocular lens; Multifocal contact lens; Visual acuity; Patient satisfaction; Contrast sensitivity; Cataract surgery; Presbyopia 


\section{Key Summary Points}

\section{Why carry out this study?}

Both multifocal intraocular (IOL) and contact lenses $(\mathrm{CL})$ have been considered among the best for clinical presbyopia correction. Nevertheless, detailed visual performance and patient satisfaction after multifocal IOL implantation and during multifocal CL wear in IOL-implanted eyes have so far not been directly compared

\section{What was learned from the study?}

The multifocal IOL and CL groups both showed good near-to-distance binocular vision and subsequent high patient satisfaction with acceptable contrast sensitivity. It is suggested that both treatments are feasible options for correcting presbyopia in older subjects

\section{INTRODUCTION}

Modern cataract surgery has been broadly recognized as refractive and presbyopia-correcting surgery. Accordingly, it is of clinical importance to precisely correct refractive errors as much as possible and to obtain good near-to-distance visual acuity even without spectacle correction to further improve visual performance and subsequent patient satisfaction. Nowadays, the refractive accuracy of cataract surgery has been considerably enhanced by introducing the latest intraocular lens (IOL) power calculation formulas and optical biometry. However, some patients do complain of their presbyopic vision nowadays, even after monofocal IOL implantation. Multifocal IOL implantation been has shown to be effective for attaining good near and far vision, thus reducing spectacle dependence of cataract patients [1-3]. Especially trifocal IOLs have been reported to provide good near, intermediate, and far vision and subsequent high patient satisfaction in recent years
[4-12]. However, some patients are dissatisfied with their vision quality, possibly due to glare, halo, starburst, refractive errors, concomitant eye diseases, or neural adaptation failure [13-15]. Actually, multifocal IOL explanation is sometimes necessary, especially when these complaints are severe and persistent over time [15]. Bifocal contact lens (CL) wear has also been demonstrated to be effective for obtaining good near-to-far visual outcomes, thus reducing spectacle dependence in presbyopic subjects [16-18]. Multifocal CLs have several advantages over multifocal IOLs in terms of a preference of non-surgical procedures, the prevention of the possible risk of postoperative complications, the reversibility, and the variability of the lens power with aging. Moreover, it can be applied a long time after cataract surgery has been performed $[19,20]$. Both multifocal IOLs and CLs have been considered one of the best technologies for presbyopic correction that are currently available in daily practice. However, to the best of our knowledge, no direct comparison of detailed visual performance and patient satisfaction after multifocal IOL implantation and during multifocal CL wear in IOL-implanted eyes has so far been conducted. It may give us intrinsic insights into the clinical implication and the patient selection of multifocal IOLs and multifocal CLs in IOL-implanted eyes. This multicenter study aims to retrospectively compare the detailed visual performance and patient satisfaction in multifocal IOL-implanted eyes and multifocal-CL wearing eyes after monofocal IOL implantation.

\section{METHODS}

\section{Study Population}

The study protocol was registered with the University Hospital Medical Information Network Clinical Trial Registry (000045132). This retrospective review of the clinical charts comprised a total of 102 eyes of 51 consecutive patients who underwent standard phacoemulsification with bilateral trifocal IOL implantation (multifocal IOL group) and 40 eyes of 20 consecutive patients who wore multifocal CLs 
after non-toric monofocal IOL implantation (multifocal CL group). Patients aged $\geq 75$ years, eyes with postoperative best-corrected visual acuity of $\geq 0.1$ logMAR, eyes with corneal astigmatism of $\geq 1.5$ diopters (D), eyes with any history of ocular surgery, ocular trauma, or other concomitant eye diseases, and eyes developing any intraoperative or postoperative complications were excluded from the study. A total of 70 and 40 patients were recruited at Miyata Eye Hospital and Kitasato University Hospital, respectively, and 51 (73\%) and 20 (50\%) patients underwent these multifocal IOL and CL treatments, respectively. This research was approved by the Institutional Review Board at Miyata Eye Hospital (CS-362-005) and followed the tenets of the Declaration of Helsinki. The Institutional Review Board waived the requirement for informed consent for this retrospective review of the clinical charts.

\section{Multifocal Intraocular Lens Implantation}

Two experienced surgeons performed standard phacoemulsification under topical anesthesia through a 2.4-mm corneoscleral incision using the same techniques, comprising capsulorhexis, nuclear and cortex extraction, and trifocal IOL (Acrysof PanOptix trifocal, Alcon Laboratories, Fort Worth, TX, USA) implantation in the capsular bag. This PanOptix trifocal IOL is a nonapodized, ultraviolet- and blue light-filtering, hydrophobic acrylic lens having a central diffractive zone of $4.5 \mathrm{~mm}$ with 15 diffractive rings that divides the incoming light to create $+2.17 \mathrm{D}$ intermediate and $+3.25 \mathrm{D}$ near add powers. It uses zeroth, second, and third diffractive orders for distance, intermediate, and near foci, respectively, and the energy at the first diffractive order is redistributed to optimize optical performance at three focal points [4-12]. Postoperatively, steroidal $(0.1 \%$ betamethasone), antibiotic (1.5\% levofloxacin), diclofenac sodium $(0.1 \%$ bromfenac $)$ medications were topically administered for 2 weeks, with the dose being steadily reduced after that.

\section{Multifocal Contact Lens Prescription}

We prescribed multifocal contact lenses (Dailies total 1 multifocal, Alcon Laboratories, Fort Worth, TX, USA) 1 month after non-toric monofocal IOL implantation performed by two experienced surgeons in the multifocal CL group. The subjects were in part comprised of those in the preceding report [19]. This Dailies total 1 bifocal CL is a silicone hydrogel, daily disposable, center-near aspheric, and progressive refractive lens (delefilcon $\mathrm{A}$ ), with three additional powers $(+1.25 \mathrm{D},+2.0 \mathrm{D}$, and + $2.5 \mathrm{D})$ for near vision in the central region and a base curve of $8.5 \mathrm{~mm}$ and CL diameter of $14.1 \mathrm{~mm}$ [21-23]. During an initial trial, the lens power was targeted at emmetropia with the lowest additional power $(+1.25 \mathrm{D})$. In addition, the spherical and additional power was individually adjusted with preliminary evaluation and determined based on patient preferences of near-to-far binocular vision.

\section{Outcome Measures}

We quantitatively assessed binocular visual acuity at all distances, contrast sensitivity (CS) function, patient satisfaction, the rate of the spectacle user, and adverse events and complications 1 month after multifocal IOL implantation and during multifocal CL wear 1 month after daily use of the multifocal CL. We determined visual acuity at $0.3,0.5,0.7,1$, and $5 \mathrm{~m}$, using a decimal acuity chart of Landolt rings shown in horizontal or vertical direction gaps under photopic conditions $(500 \mathrm{l} \times)$. We also determined CS function using a contrast sensitivity unit (VCTS-6500, Vistech) with the best spectacle correction at $2.5 \mathrm{~m}$, at 3, 6, 12, and 18 cycles, under the same conditions. Finally, we calculated the area under the log contrast sensitivity function (AULCSF) from the obtained CS data, as described previously [24]. In brief, the $\log$ of CS was plotted as a function of log spatial frequency, and third-order polynomials were fitted to the data. The fitting curve was 
integrated, and the resultant value was defined as the AULCSF. We also evaluated the patient satisfaction score for the overall vision, using a visual analogue scale (VAS) with symptom intensity scores on a scale of 0 (no satisfaction) to 100 (maximum satisfaction), the rate of spectacle users, and subjective symptoms such as glare or halos in daily activities using a questionnaire in both groups. All examinations were conducted by experienced ophthalmic technicians who were masked to the clinical condition of the subjects and the preliminary results of the study.

\section{Statistical Analysis}

We first checked the normality of all data samples by the Shapiro-Wilk test using statistical software (Bellcurve for Excel, Social Survey Research Information Co, Ltd., Tokyo, Japan). Because all data did not fulfill the criteria for normal distribution, the Mann-Whitney $U$ test was applied to compare the biometric data between the two groups. Fisher's exact test was used to compare the percentages between the two groups. Unless otherwise indicated, the results are expressed as mean \pm standard deviation, and a value of $p<0.05$ was deemed statistically significant.

\section{RESULTS}

Table 1 shows the baseline demographics of the study population. All surgeries were uneventful, and no intraoperative complications occurred in the multifocal IOL group. In the multifocal CL group, eyes with additional powers of + 2.00 and $+2.50 \mathrm{D}$ were 32 eyes $(80 \%)$ and 8 eyes (20\%). Binocular visual acuity at 0.3, 0.5, $0.7,1$, and $5 \mathrm{~m}$ was $0.05 \pm 0.11,-0.02 \pm 0.09$, $-0.02 \pm 0.09, \quad-0.02 \pm 0.09, \quad$ and $-0.04 \pm 0.07$, respectively, in the multifocal IOL group and $0.25 \pm 0.13,0.04 \pm 0.10$, $0.01 \pm 0.09, \quad-0.01 \pm 0.07$, and $0.00 \pm 0.08$, respectively, in the multifocal CL group (Fig. 1). We found significant differences in binocular acuity at $30 \mathrm{~cm}(p<0.001), 50 \mathrm{~cm}(p<0.001)$, $70 \mathrm{~cm}(p=0.007), 1 \mathrm{~m}(p=0.017)$, and $5 \mathrm{~m}$ $(p<0.001)$. Figure 2 shows CS function according to $3,6,12$, and 18 cycles/degree. We found no significant differences at 6,12 , and 18 cycles/degree ( $p=0.931,0.800$, and 0.055$)$, but a significant difference at 3 cycle/degree $(p=0.002)$. The AULSCSF was $1.32 \pm 0.14$ and $1.33 \pm 0.16$ in the multifocal IOL and CL groups, respectively $(p=0.444)$. The patient satisfaction score for overall visual performance significantly improved, from $25.5 \pm 22.9$ preoperatively to $80.2 \pm 20.6$ postoperatively in the multifocal IOL group $(p<0.001)$ and significantly improved from $55.9 \pm 26.0$ before CL

Table 1 Preoperative demographics of the study population

\begin{tabular}{lllr}
\hline Demographics & Multifocal IOL group & Multifocal CL group & $\boldsymbol{p}$ value \\
\hline Eyes & 102 & 40 & \\
Age (years) & $63.6 \pm 8.6$ & $59.3 \pm 9.8$ & 0.061 \\
Gender (male:female) & $21: 30$ & $4: 16$ & 0.107 \\
UCVA (logMAR) & $0.81 \pm 0.59$ & $0.64 \pm 0.34$ & 0.393 \\
BSCVA (logMAR) & $0.16 \pm 0.39$ & $-0.16 \pm 0.08$ & $<0.001$ \\
Manifest spherical equivalent (D) & $-2.88 \pm 4.55$ & $-2.13 \pm 0.92$ & 0.212 \\
Mean keratometric readings (D) & $44.15 \pm 1.35$ & $44.02 \pm 1.44$ & 0.946 \\
Axial length (mm) & $24.66 \pm 1.66$ & $27.05 \pm 1.54$ & $<0.001$ \\
\hline
\end{tabular}

$I O L$ intraocular lens, $C L$ contact lens, $\log M A R$ logarithm of the minimal angle of resolution, $U C V A$ uncorrected visual acuity, $B S C V A$ best spectacle-corrected visual acuity, $D$ diopter 


\section{$-0.20$}

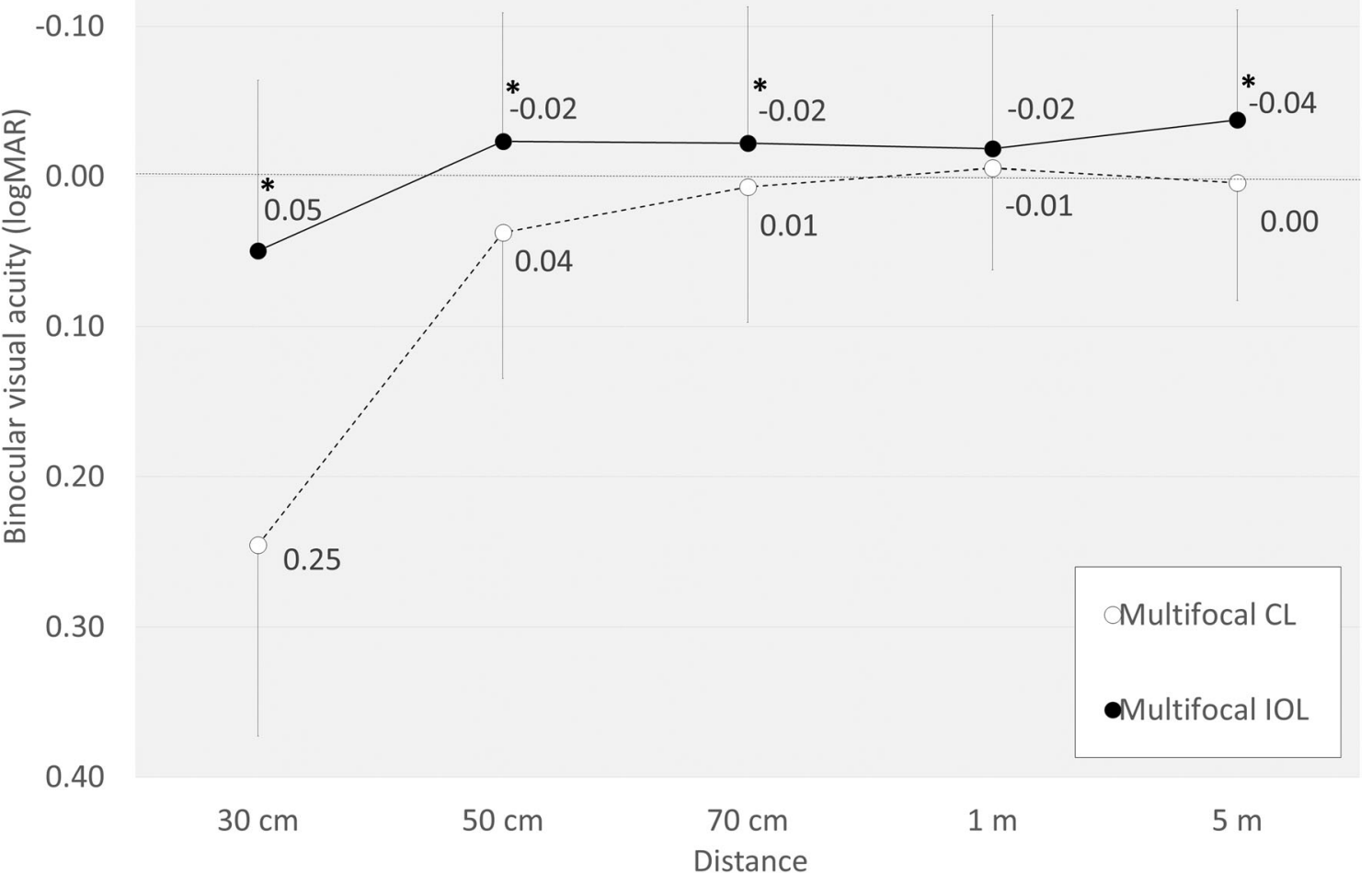

Fig. 1 Graph showing binocular visual acuity at all distances $(0.3,0.5,0.7,1$, and $5 \mathrm{~m})$ in the multifocal intraocular lens $(\mathrm{IOL})$ and multifocal contact lens $(\mathrm{CL})$ groups

wear to $82.9 \pm 10.5$ during CL wear in the multifocal CL group $(p<0.001)$. We found a significant difference in the pre-treated satisfaction $(p<0.001)$ but no significant differences in the post-treated satisfaction between the two groups $(p=0.889)$. The rate of spectacle users in daily activities significantly decreased, from 94.1 to $23.4 \%$ in the multifocal IOL group $(p<0.001)$, and significantly decreased, from 90.0 to $41.7 \%$, in the multifocal CL group $(p<0.001)$. We also found no significant differences in the post-treated rate of spectacle users between the two groups $(p=0.277)$.

We found mild subjective symptoms such as glare or halos in 35 eyes (69\%) and 24 eyes $(60 \%)$ in the multifocal IOL and CL groups, respectively $(p=0.507)$. Only one eye $(2.0 \%)$ had a moderate-to-severe visual disturbance at night in the multifocal IOL group, but this gradually recovered over time without secondary interventions. Otherwise, we found no postoperative complications such as infectious keratitis, posterior capsular opacification, cystoid macular edema, or waxy vision during the observation period.

\section{DISCUSSION}

In the current study, our findings showed that both multifocal IOLs and CLs provided good near-to-distance binocular vision and subsequent high patient satisfaction for overall vision in daily activities but that CS function slightly decreased. Still, all eyes were acceptable and within their normal range at all spatial frequencies. Thus, our results may support the 


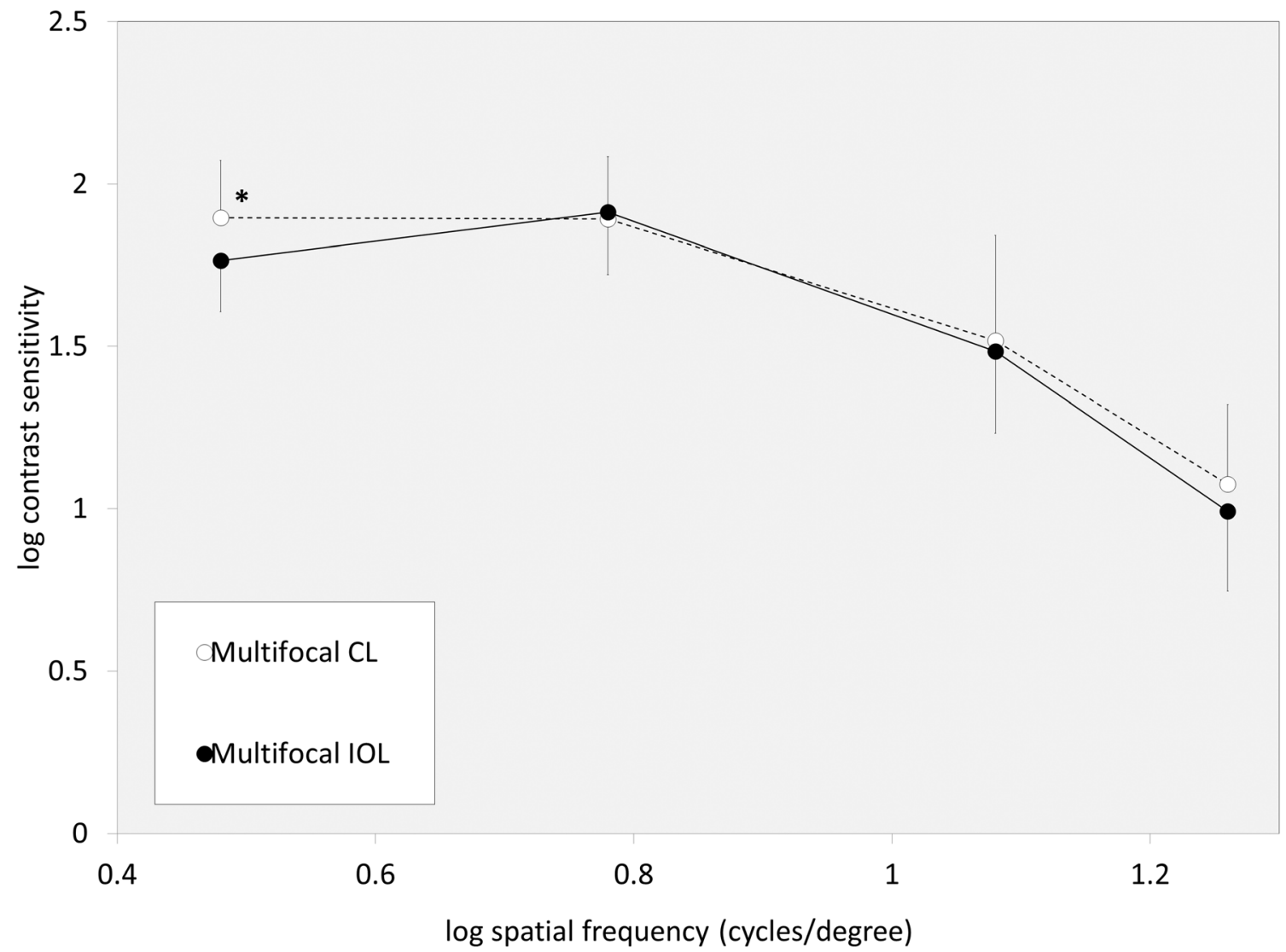

Fig. 2 Graph showing contrast sensitivity (CS) function in the multifocal intraocular lens (IOL) and multifocal contact lens $(\mathrm{CL})$ groups

view that both presbyopic treatments are feasible options for correcting presbyopia in elderly subjects. As far as we can ascertain, this is the first published study to directly compare the detailed visual performance and patient satisfaction between multifocal IOL-implanted eyes and multifocal CL-wearing eyes after monofocal IOL implantation. Although we accept that the patient backgrounds such as age and presbyopia-correcting technology (diffractive trifocal vs. refractive bifocal) were not well matched between the two groups, we believe that this information is simple but clinically helpful for comparing the best technology and grasping the real-world condition of such promise presbyopia-correcting treatments. Moreover, it will be beneficial for predicting overall visual acuity at near-to-far distances and CS function after both presbyopia-correcting treatments. We previously stated that middle-aged female patients with contact lens wear experience to correct myopia might be good candidates for multifocal CL wear after cataract surgery [20]. Originally high myopic patients before cataract surgery tended to select the multifocal CL treatment in the current study, presumably because such patients were accustomed to wearing CLs in their daily lifes. Therefore, we assume that the preoperative axial length was significantly larger in the multifocal CL group than that in the multifocal IOL group. We performed multifocal IOL implantation due to the presence of cataracts preoperatively in all eyes. Since these two groups have different motivations and expectations concerning presbyopic correction, we assume that it is difficult to completely match these baseline demographics in a clinical setting. 
Conventional bifocal IOLs have two focus points at near and far and thus provide good near and distant vision without spectacle correction in cataract patients. However, considering the prevalence of visual display terminals such as smartphones and personal computers, intermediate vision plays an essential role in overall optical performance in daily activities nowadays. Therefore, trifocal IOLs have recently been developed to rectify such drawbacks of bifocal IOL to improve intermediate vision and thus have been reported to be effective for continuously attaining near-to-far vision without spectacle correction [4-12]. Nevertheless, multifocal IOL explantation and subsequent monofocal IOL implantation were rare but sometimes necessary, possibly because of a loss of CS function such as waxy vision, photic phenomena, or neural adaptation failure [6]. Multifocal CLs have advantages over multifocal IOLs, especially in terms of reversibility. Therefore, it is easier to stop wearing multifocal CLs when the patients complain about their vision.

Table 2 summarizes previous studies on visual acuity at all distances of the trifocal IOLs and bifocal CLs used in the present study. Our results were in good agreement with earlier studies in that both multifocal IOLs and CLs provided good near, intermediate, and far visual outcomes without spectacle correction $[4-12,19,20]$. Thus, it is indicated that both multifocal IOL and CL treatments are viable alternatives for presbyopic correction in elderly patients who wish to reduce the chance of spectacle correction. However, it should be noted that multifocal IOLs showed slightly but significantly better visual outcomes, especially at a near distance in this study population. These findings indicate that trifocal IOLs may be slightly superior to bifocal CLs in visual acuity at all distances. However, these visual differences were essentially small, especially at a far distance. Interestingly, most patients in the multifocal CL group selected the CL with an additional power of $+2.0 \mathrm{D}$ for near vision, presumably because a CL with an additional power of $+2.5 \mathrm{D}$ might decrease CS function and might not be acceptable for daily use in IOL-implanted eyes.
Both multifocal IOLs and CLs also induced a slight deterioration of CS function, especially at a high spatial frequency, in line with previous studies on CS after trifocal IOL implantation and bifocal CL wear. Nevertheless, all eyes were within the normal range at all spatial frequencies. Therefore, we assume that CS was still clinically acceptable for this kind of presbyopic treatment, and thus the post-treatment satisfaction scores were overall high in both groups. Indeed, there are ongoing concerns about the loss of CS after diffractive multifocal IOL implantation and during refractive multifocal CL wear. Still, it has been demonstrated that trifocal IOLs are almost comparable to monofocal IOLs in terms of CS function, possibly because they are designed to effectively use $88 \%$ of the available light intensity $[8-10,12]$. Furthermore, it has been reported that wearing bifocal CLs slightly reduced CS at high spatial frequencies, but that CS function remained within the normal range in these eyes $[19,20]$. Thus, our findings were in good agreement with all previous studies on CS function after trifocal IOL implantation and during bifocal CL wear in IOL-implanted eyes.

There are several limitations to this study. First, this research was conducted in a retrospective fashion, and thus some baseline characteristics such as BSCVA and axial length were somewhat different between the two groups. Hence, these background differences can be possible sources of selection bias. However, this study may refract the actual status of visual quality and patient satisfaction in the presbyopic population in a clinical setting. Second, multifocal IOLs have applied a diffractive trifocal technology, which is essentially different from the refractive bifocal technology involved in multifocal CLs. Nevertheless, we have used one of the best clinically available technologies for presbyopic correction in daily practice. Third, we did not measure pupil diameter or visual performance under mesopic conditions in this study population. We await further studies to understand the role of pupil size in visual performance and the detailed visual performance with different light conditions in multifocal IOL-implanted eyes and during multifocal CL wear. Fourth, we did not evaluate 


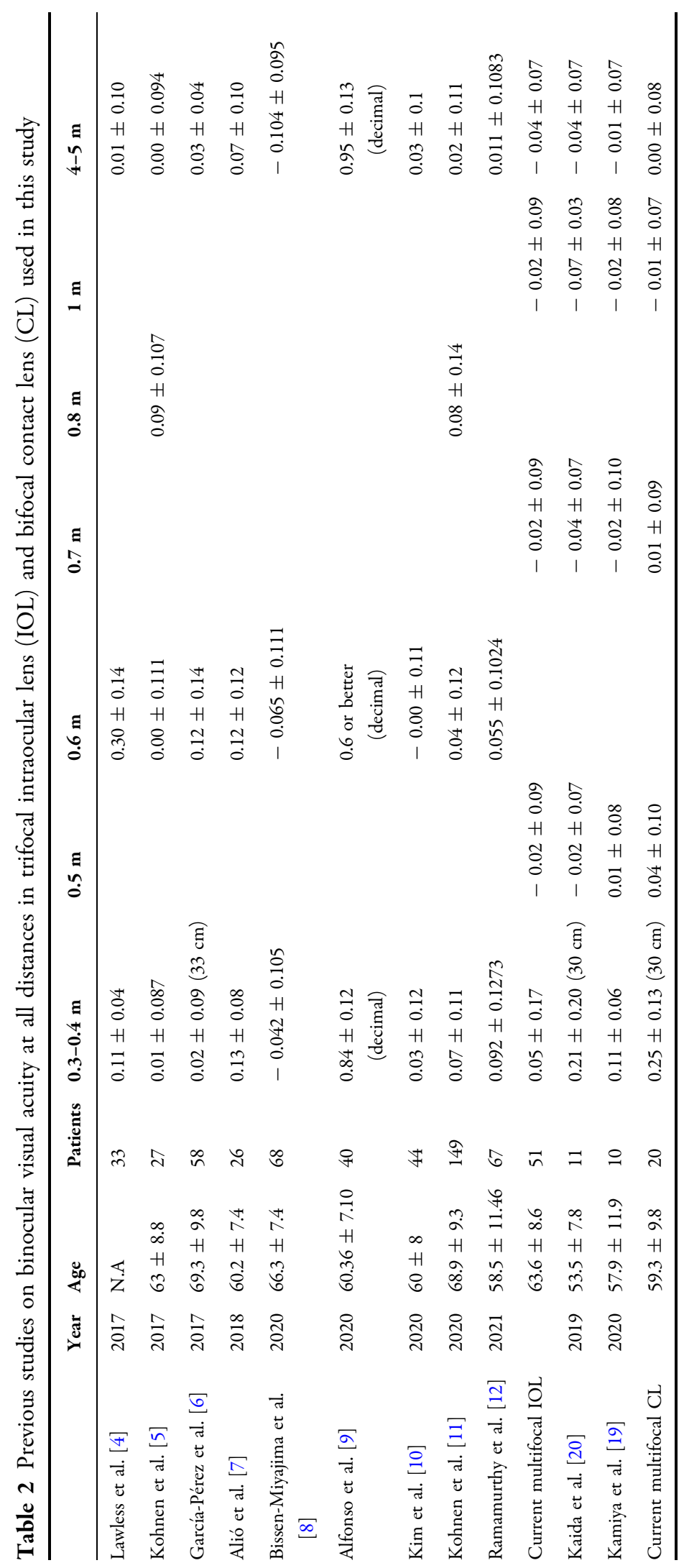


the detailed defocus curves of the two lenses nor did we use a validated questionnaire to assess subjective symptoms.

\section{CONCLUSION}

In summary, our findings confirmed that both multifocal IOLs and CLs provided good near-tofar vision and subsequent high patient satisfaction, with proper CS function, even though its function slightly decreased, suggesting that both multifocal IOLs and CLs may be feasible treatment options for presbyopic correction. However, it should be noted that the detailed visual performance in multifocal IOL-implanted eyes was slightly better than that during multifocal CL wear in monofocal IOL-implanted eyes. Based on our findings, we believe that this information is clinically helpful for understanding the clinical implication and patient selection of presbyopic correction methods in older subjects that wish to reduce the chance of spectacle correction.

\section{ACKNOWLEDGEMENTS}

We thank all study participants for their involvement in the study.

Funding. This research was supported in part by an unrestricted investigator-initiated grant from Alcon Japan Ltd. The funders had no role in study design, data collection and analysis, decision to publish, or preparation of the manuscript.

Authorship. All named authors meet the International Committee of Medical Journal Editors (ICMJE) criteria for authorship for this article, take responsibility for the integrity of the work as a whole, and have given their approval for this version to be published.

Author Contributions. Kazutaka Kamiya and Kazunori Miyata were involved in the design and conduct of the study, Kazutaka Kamiya, Kei Iijima, Keita Takada, and Yosai Mori were involved in the collection, management, analysis, and interpretation of data, and all authors were involved in preparation, review, and final approval of the manuscript.

Disclosures. Kazutaka Kamiya, Kei Iijima, Takahiro Hiraoka, Yosai Mori, and Kazunori Miyata declare that they have nothing to disclose.

Compliance with Ethics Guidelines. This review was approved by the Institutional Review Board of Miyata Eye Hospital (CS-362005) and followed the tenets of the Declaration of Helsinki. The Institutional Review Board waived the requirement for informed consent for this retrospective review of the clinical charts.

Data Availability. The datasets analysed during the current study are available from the corresponding author on reasonable request.

Open Access. This article is licensed under a Creative Commons Attribution-NonCommercial 4.0 International License, which permits any non-commercial use, sharing, adaptation, distribution and reproduction in any medium or format, as long as you give appropriate credit to the original author(s) and the source, provide a link to the Creative Commons licence, and indicate if changes were made. The images or other third party material in this article are included in the article's Creative Commons licence, unless indicated otherwise in a credit line to the material. If material is not included in the article's Creative Commons licence and your intended use is not permitted by statutory regulation or exceeds the permitted use, you will need to obtain permission directly from the copyright holder. To view a copy of this licence, visit http://creativecommons.org/licenses/by$\mathrm{nc} / 4.0 /$.

\section{REFERENCES}

1. de Silva SR, Evans JR, Kirthi V, Ziaei M, Leyland M. Multifocal versus monofocal intraocular lenses after 
cataract extraction. Cochrane Database Syst Rev. 2016;12:CD003169.

2. Cao K, Friedman DS, Jin S, et al. Multifocal versus monofocal intraocular lenses for age-related cataract patients: a system review and meta-analysis based on randomized controlled trials. Surv Ophthalmol. 2019;64:647-58.

3. Negishi K, Hayashi K, Kamiya K, Sato M, BissenMiyajima H, Survey Working Group of the Japanese Society of Cataract and Refractive Surgery. Nationwide prospective cohort study on cataract surgery with multifocal intraocular lens implantation in Japan. Am J Ophthalmol. 2019;208:133-44.

4. Lawless M, Hodge C, Reich J, et al. Visual and refractive outcomes following implantation of a new trifocal intraocular lens. Eye Vis (Lond). 2017;4:10.

5. Kohnen T, Herzog M, Hemkeppler E, et al. Visual performance of a quadrifocal (trifocal) intraocular lens following removal of the crystalline lens. Am J Ophthalmol. 2017;184:52-62.

6. García-Pérez JL, Gros-Otero J, Sánchez-Ramos C, Blázquez V, Contreras I. Short term visual outcomes of a new trifocal intraocular lens. BMC Ophthalmol. 2017;17:72.

7. Alió JL, Plaza-Puche AB, Alió Del Barrio JL, et al. Clinical outcomes with a diffractive trifocal intraocular lens. Eur J Ophthalmol. 2018;28: 419-24.

8. Bissen-Miyajima H, Ota Y, Hayashi K, Igarashi C, Sasaki N. Results of a clinical evaluation of a trifocal intraocular lens in Japan. Jpn J Ophthalmol. 2020;64:140-9.

9. Alfonso JF, Fernández-Vega-Cueto L, FernándezVega L, Montés-Micó R. Visual function after implantation of a presbyopia-correcting trifocal intraocular lens. Ophthalmic Res. 2020;63:152-64.

10. Kim TI, Chung TY, Kim MJ, Lee K, Hyon JY. Visual outcomes and safety after bilateral implantation of a trifocal presbyopia correcting intraocular lens in a Korean population: a prospective single-arm study. BMC Ophthalmol. 2020;20:288.

11. Kohnen T, Marchini G, Alfonso JF, et al. Innovative trifocal (quadrifocal) presbyopia-correcting IOLs: 1-year outcomes from an international multicenter study. J Cataract Refract Surg. 2020;46:1142-8.

12. Ramamurthy D, Vasavada A, Padmanabhan P, et al. Clinical outcomes after bilateral implantation of a trifocal presbyopia-correcting intraocular lens in an Indian population. Clin Ophthalmol. 2021;15: 213-25.
13. Woodward MA, Randleman JB, Stulting RD. Dissatisfaction after multifocal intraocular lens implantation. J Cataract Refract Surg. 2009;35: 992-7.

14. de Vries NE, Webers CA, Touwslager WR, et al. Dissatisfaction after implantation of multifocal intraocular lenses. J Cataract Refract Surg. 2011;37: 859-65.

15. Kamiya K, Hayashi K, Shimizu K, Negishi K, Sato M, Bissen-Miyajima H, Survey Working Group of the Japanese Society of Cataract and Refractive Surgery. Multifocal intraocular lens explantation: a case series of 50 eyes. Am J Ophthalmol. 2014;158: 215-20.

16. Bennett ES. Contact lens correction of presbyopia. Clin Exp Optom. 2008;91:265-78.

17. Pérez-Prados R, Piñero DP, Pérez-Cambrodí RJ, Madrid-Costa D. Soft multifocal simultaneous image contact lenses: a review. Clin Exp Optom. 2017;100:107-27.

18. Wolffsohn JS, Davies LN. Presbyopia: effectiveness of correction strategies. Prog Retin Eye Res. 2019;68:124-43.

19. Kamiya K, Fujimura F, Ando W, Iijima K, Shoji N. Visual performance and patient satisfaction of multifocal contact lenses in eyes undergoing monofocal intraocular Lens implantation. Contact Lens Anterior Eye. 2020;43:218-21.

20. Kaida T, Yukawa C, Higashi S, Minami K, Miyata K. Presbyopia correction using multifocal soft contact lenses in patients with monofocal intraocular lenses. Eye Contact Lens. 2020;46:234-7.

21. Plainis S, Atchison DA, Charman WN. Power profiles of multifocal contact lenses and their interpretation. Optom Vis Sci. 2013;90:1066-77.

22. Montés-Micó R, Madrid-Costa D, Domínguez-Vicent A, Belda-Salmerón L, Ferrer-Blasco T. In vitro power profiles of multifocal simultaneous vision contact lenses. Contact Lens Anterior Eye. 2014;37: $162-7$.

23. Kim E, Bakaraju RC, Ehrmann K. Power profiles of commercial multifocal soft contact lenses. Optom Vis Sci. 2017;94:183-96.

24. Applegate RA, Howland HC, Sharp RP, Cottingham AJ, Yee RW. Corneal aberrations ad visual performance after radial keratotomy. J Refract Surg. 1998;14:397-407. 